\title{
Influence of meal intake on pulse wave indices in type 2 diabetes
}

\author{
Norikatsu Morioka ${ }^{1}$, Jun-ichi Funada ${ }^{1}$, Yasunori Takata ${ }^{2}$, Hidetoshi Hashida ${ }^{1}$, Takeru Iwata ${ }^{1}$, Jitsuo Higaki ${ }^{3}$ \\ and Hideki Okayama ${ }^{3}$
}

Augmentation index (AI), brachial-ankle pulse wave velocity (baPWV) and cardio-ankle vascular index (CAVI) are available for the assessment of arterial stiffness in clinical practices. However, influences of meal intake on these indices are still poorly understood. The aim of this study is to elucidate the effects of daily meal intake on pulse wave indices in patients with type 2 diabetes. We studied 17 patients with type 2 diabetes. Al was measured at fasting, 60 and 120 min after a commercial mixed meal $(500 \mathrm{kcal})$ intake. The baPWV and CAVI were measured at fasting and $80-100 \mathrm{~min}$ after meal intake. All pulse indices decreased significantly after meal intake (Al, $89.3 \pm 9.7 \%$ to $77.9 \pm 9.4 \%, 82.0 \pm 8.4 \%, P<0.001$; baPWV, $1652 \pm 286$ $1586 \pm 240 \mathrm{~cm} \mathrm{~s}^{-1}, P=0.002$; CAVI, $\left.9.52 \pm 0.92-9.20 \pm 0.89, P=0.037\right) . \Delta_{120}$ (value $120 \mathrm{~min}$ after meal intake-fasting value) Al correlated significantly with age, body weight, $\Delta_{120}$ systolic blood pressure (SBP), $\Delta_{120}$ diastolic blood pressure, $\Delta_{120}$ pulse pressure, $\Delta_{120}$ heart rate and fasting AI. $\Delta$ (postprandial value-fasting value) baPWV correlated significantly with fasting baPWV, $\Delta$ SBP, $\Delta$ pulse pressure and HbA1c. In contrast, $\Delta$ CAVI did not correlate with any clinical variables. In conclusion, postprandial decreases in AI, baPWV and CAVI can lead to underestimate arterial stiffness in patients with type 2 diabetes. Postprandial changes in Al and baPWV, but not CAVI, are associated with changes in hemodynamic variables after daily meal intake.

Hypertension Research (2010) 33, 743-747; doi:10.1038/hr.2010.66; published online 30 April 2010

Keywords: augmentation index; cardio-ankle vascular index; diabetes mellitus; postprandial change; pulse wave velocity

\section{INTRODUCTION}

Aortic stiffness is an independent predictor of all-cause and cardiovascular mortality in patients with hypertension, ${ }^{1,2}$ diabetes mellitus ${ }^{3}$ and end-stage renal disease. ${ }^{4,5}$ Aortic pulse wave velocity (PWV) has been widely used to assess arterial stiffness. However, measurements of aortic PWV are technically difficult because it necessitates the use of two oscillometric sensors positioned over the left common carotid artery and the left femoral artery. Recently, other indices such as the brachial-ankle PWV (baPWV) and the cardio-ankle vascular index (CAVI) have been developed in Japan as alternative parameters for arterial stiffness. ${ }^{6-8}$ Earlier studies have shown that baPWV and CAVI reflect atherosclerosis. ${ }^{7-10}$ In addition, the augmentation index (AI) of radial artery, which provides information on central blood pressure, ${ }^{11-13}$ has been developed as arterial stiffness parameter. ${ }^{14,15} \mathrm{AI}$ has been also reported to be associated with severity of atherosclerosis and coronary risk factors. ${ }^{16,17}$ Accordingly, AI, baPWV and CAVI have been used in routine examinations because of the convenience with which their measurements can be taken.

Diabetes mellitus is a major risk factor for atherosclerosis. In fact, patients with type 2 diabetes have been reported to have a two- to fourfold increased risk of cardiovascular mortality, ${ }^{18}$ and accordingly, assessment of aortic stiffness has been widely accepted as an important parameter in risk stratification for patients with diabetes mellitus. However, clinical examinations are often performed in postprandial state as well as fasting state, especially in outpatient clinics. On the other hand, postprandial effects on these pulse wave indices are still poorly understood. Therefore, the aim of this study is to elucidate the effects of daily meal intake on these pulse wave indices in patients with type 2 diabetes.

\section{METHODS}

Study patients

We studied 17 patients with type 2 diabetes as diagnosed by the criteria of the American Diabetes Association. ${ }^{19}$ Patients under insulin therapy or those who had suffered from heart failure (left ventricular ejection fraction $<50 \%$ ), atrial fibrillation, renal dysfunction (serum creatinine level $>1.5 \mathrm{mg}$ per $100 \mathrm{ml}$ ) or peripheral artery disease (ankle-brachial index $<0.9$ ) were excluded from this study. This study was performed in accordance with the Declaration of Helsinki (1989) of the World Medical Association and was approved by the ethics committee of National Hospital Organization Ehime Hospital. All subjects gave written informed consent to participate in this study.

${ }^{1}$ Department of Cardiology, National Hospital Organization Ehime Hospital, Toon, Japan; ${ }^{2}$ Department of Molecular and Genetic Medicine, Ehime University Graduate School of Medicine, Toon, Japan and ${ }^{3}$ Division of Cardiology, Department of Integrated Medicine and Informatics, Ehime University Graduate School of Medicine, Toon, Japan Correspondence: Dr J Funada, Department of Cardiology, National Hospital Organization Ehime Hospital, 366 Yokogawara, Toon, Ehime 791-0281, Japan. E-mail: jfunada@ehime-nh.go.jp

Received 27 November 2009; revised 18 February 2010; accepted 17 March 2010; published online 30 April 2010 


\section{Study protocol}

After overnight fasting, all patients ingested a commercial mixed meal (CalorieMate 500 kcal: carbohydrate $51 \mathrm{~g}$, fat $28 \mathrm{~g}$, protein $10 \mathrm{~g}$; Otsuka Pharmaceutical, Tokyo, Japan) with $300-500 \mathrm{ml}$ of water within $15 \mathrm{~min}$. Measurements of $\mathrm{AI}$ and blood samplings were performed during the fast, as well as 60 and $120 \mathrm{~min}$ after the meal intake. CAVI and baPWV were measured during the fast and $80-100 \mathrm{~min}$ after the meal intake. The administration of antihypertensive drugs was prohibited at least $24 \mathrm{~h}$ before the study. All medications that affect lipid metabolism were withheld for at least $72 \mathrm{~h}$ before the study. Patients were also advised not to take any antidiabetic drugs from the evening before the study until the study was completed.

\section{Measurement of radial AI}

After 10 min of rest in a sitting position, systolic blood pressure (SBP) and diastolic blood pressure (DBP) were measured at the right brachial artery. A pulse waveform of the left radial artery was recorded by an automated tonometric system (HEM-9000AI; Omron Healthcare, Kyoto, Japan). AI was calculated as follows: $\mathrm{AI}=($ second peak $\mathrm{SBP}-\mathrm{DBP}) /($ first peak $\mathrm{SBP}-\mathrm{DBP})$ $\times 100(\%)$. This calculation was performed using a fourth-order differential equation for the radial arterial waveform. Detailed procedure about this device was reported in our earlier study. ${ }^{13}$ The coefficients of variation for intra- and inter-observer measurements of AI were $4.3 \pm 3.0$ and $2.5 \pm 1.7 \%$, respectively. ${ }^{13}$

\section{Measurement of baPWV}

The patients were placed in the supine position for at least $5 \mathrm{~min}$, and then ECG and heart sound were monitored. Cuffs were wrapped around both upper arms and ankles. BaPWV was measured using an automatic waveform analyzer (form PWV/ABI, BP-203RPE II; Colin, Komaki, Japan) according to the following equation: baPWV $=(D 1-D 2) / T 1$, where $D 1$ is the distance from the heart to the ankle and $D 2$ is the distance from the heart to the right upper arm. These distances were calculated on the basis of the patient's height. T1 is the time between the onset of the rise in the pulse volume record of the right upper arm and the onset of the rise in the pulse volume record of the left ankle. The average coefficient of variation for the measurement of baPWV was reported to be $\leqslant 10.0 \%{ }^{7}$

\section{Measurement of CAVI}

After measurement of baPWV, CAVI was measured using an automatic vascular screening system (VaSera VS-1000; Fukuda Denshi, Tokyo, Japan). PWV was obtained by dividing the vascular length by the time it took for the pulse wave to propagate from the aortic valve to the ankle. SBP and DBP were measured at the brachial artery. The formula used to calculate CAVI is as follows: $\mathrm{CAVI}=a\left\{(2 \rho / \Delta P) \times \ln (\mathrm{SBP} / \mathrm{DBP}) \mathrm{PWV}^{2}\right\}+b$, where $\Delta P$ is SBP $-\mathrm{DBP}$, $\rho$ is blood density, and $a$ and $b$ are scale conversion constants to match aortic PWV. The principle of CAVI was reported earlier. ${ }^{8}$ This equation is derived from Bramwell-Hill's equation and the stiffness parameter $\beta$, and CAVI is adjusted for blood pressure based on the stiffness parameter $\beta$. Therefore, CAVI reflects the stiffness of the aorta, femoral artery and tibial artery as a whole, and theoretically, it is not affected by blood pressure. All these measurements and calculations were performed automatically in VaSera VS-1000. The average coefficient of variation for the measurement of CAVI was reported to be $<5.0 \%{ }^{8}$

\section{Statistical analysis}

All data are expressed as the mean \pm s.d. The paired Student's $t$-test or repeated measure analysis of variance was used to compare continuous data. Relations between continuous variables were analyzed by linear regression analysis. Stepwise multiple regression analysis was used to evaluate the independent determinants of $\Delta$ (postprandial value-fasting value) AI and $\Delta$ baPWV. All statistical analyses were performed using SPSS version 16.0J for Windows (SPSS Japan Inc., Tokyo, Japan) and a $P$-value $<0.05$ was considered statistically significant.

\section{RESULTS}

\section{Patient characteristics}

Table 1 shows the characteristics of the study patients. All patients were taking antidiabetic drugs, including pioglitazone (all patients), $\alpha$-glucosidase inhibitors (10 patients) and sulfonylurea (8 patients). Thirteen patients $(76.5 \%)$ were taking antihypertensive drugs, including calcium antagonists (10 patients), $\beta$-blockers ( 7 patients) and angiotensin II receptor blockers/angiotensin-converting enzyme inhibitors (7 patients). Eight patients $(47.1 \%)$ were taking statins, and one patient $(5.9 \%)$ was a smoker at the time.

\section{Effect of meal intake on clinical variables}

Table 2 shows the effects of meal intake on clinical variables. SBP, DBP and pulse pressure decreased significantly, whereas heart rate increased after meal intake. Glucose and insulin levels increased significantly after meal intake.

\section{Effect of meal intake on pulse wave indices}

AI decreased significantly after meal intake $(89.3 \pm 9.7 \%$ to $77.9 \pm 9.4 \%, 82.0 \pm 8.4 \% ; P<0.001$ ) (Figure 1a). Both baPWV and

Table 1 Baseline characteristics of patients with type 2 diabetes

\section{Variables}

\section{$N$ (male/female)}

$17(10 / 7)$

Age (years)

$66.8 \pm 4.8$

Body height $(\mathrm{m})$

$1.58 \pm 0.09$

Body weight $(\mathrm{kg})$

$58.4 \pm 7.8$

BMI $\left(\mathrm{kg} \mathrm{m}^{-2}\right)$

$23.3 \pm 3.0$

$\mathrm{SBP}(\mathrm{mm} \mathrm{Hg})$

$134.0 \pm 15.6$

$\mathrm{DBP}(\mathrm{mm} \mathrm{Hg})$

$69.3 \pm 9.2$

Pulse pressure $(\mathrm{mm} \mathrm{Hg})$

$64.7 \pm 11.4$

Heart rate (beats per minute)

$63.2 \pm 8.5$

Fasting glucose (mg per $100 \mathrm{ml}$ )

$116.4 \pm 16.3$

Fasting insulin $\left(\mu \cup \mathrm{ml}^{-1}\right)$

$4.08 \pm 1.63$

HbAlc (\%)

Total cholesterol (mg per $100 \mathrm{ml}$ )

$5.97 \pm 0.62$

Triglyceride (mg per $100 \mathrm{ml}$ )

$205.4 \pm 28.1$

$112.1 \pm 53.0$

HDL-C (mg per $100 \mathrm{ml}$ )

$66.1 \pm 16.0$

Creatinine (mg per $100 \mathrm{ml}$ )

$0.76 \pm 0.19$

Al (\%)

$89.3 \pm 9.7$

BaPWV $\left(\mathrm{cm} \mathrm{s}^{-1}\right)$

$1652 \pm 286$

CAV

$9.52 \pm 0.92$

Abbreviations: Al, augmentation index; BaPWV, brachial-ankle pulse wave velocity; BMI, body mass index; CAVI, cardio-ankle vascular index; DBP, diastolic blood pressure; HDL-C, highdensity lipoprotein-cholesterol; SBP, systolic blood pressure.

Table 2 Postprandial changes in clinical variables in patients with type 2 diabetes

\begin{tabular}{lccc}
\hline & & \multicolumn{2}{c}{ Postprandial } \\
\cline { 3 - 4 } Variables & Fasting & $60 \mathrm{~min}$ & $120 \mathrm{~min}$ \\
\hline SBP (mm Hg) & $134.0 \pm 15.6$ & $122.2 \pm 14.4^{*}$ & $123.2 \pm 13.4^{*}$ \\
DBP (mm Hg) & $69.3 \pm 9.2$ & $62.4 \pm 9.2^{*}$ & $62.7 \pm 9.8^{*}$ \\
Pulse pressure (mm Hg) & $64.7 \pm 11.4$ & $59.9 \pm 10.0^{*}$ & $60.5 \pm 9.8^{*}$ \\
Heart rate (beats per minute) & $63.2 \pm 8.5$ & $73.0 \pm 11.6^{*}$ & $66.9 \pm 9.6^{* \dagger}$ \\
Glucose (mg per 100 ml) & $116.4 \pm 16.3$ & $168.3 \pm 35.7^{*}$ & $172.5 \pm 43.1^{*}$ \\
Insulin ( $\mu \mathrm{U} \mathrm{ml} \mathrm{ml}^{-1}$ ) & $4.08 \pm 1.63$ & $15.97 \pm 7.18^{*}$ & $19.42 \pm 9.02^{* \dagger}$
\end{tabular}

Abbreviations: DBP, diastolic blood pressure; SBP, systolic blood pressure. ${ }^{\star} P<0.05$ vs. fasting; ${ }^{\dagger} P<0.05$ vs. $60 \mathrm{~min}$. 

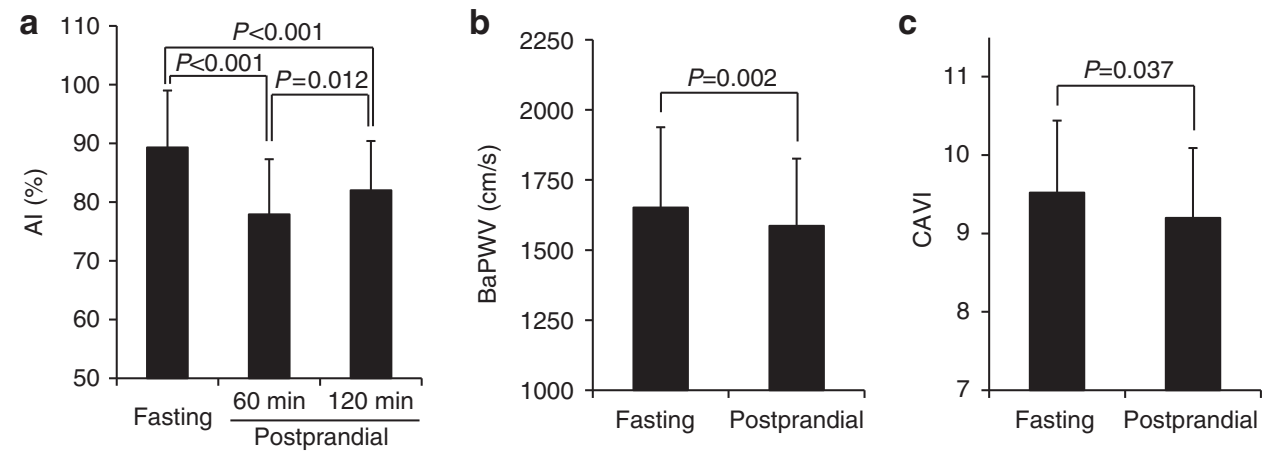

Figure 1 Comparisons between fasting and postprandial values of $\mathrm{Al}(\mathbf{a}), \mathrm{baPWV}(\mathbf{b})$ and CAVI (c) in patients with type 2 diabetes.
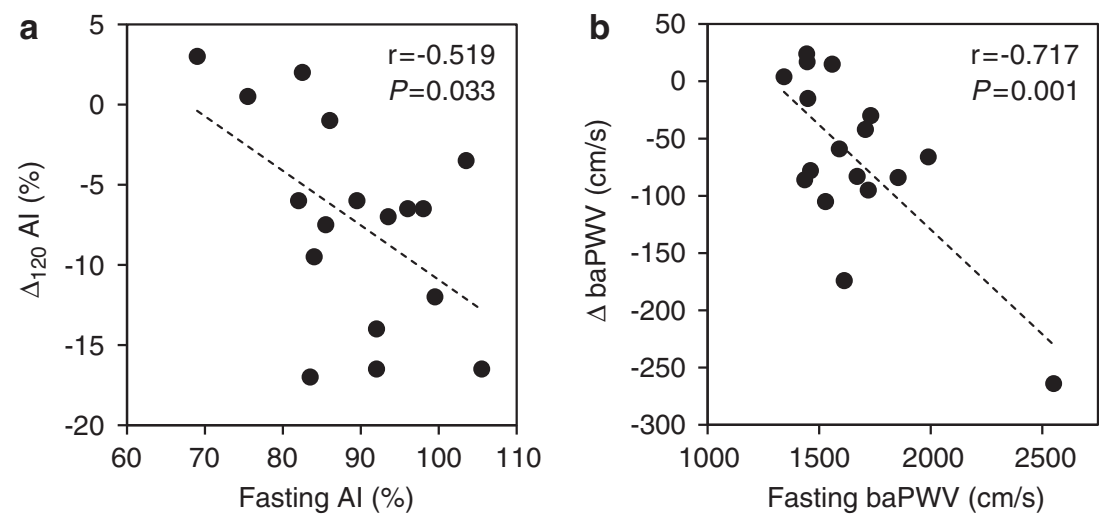

Figure 2 Relationships between the degree of postprandial changes and fasting values in Al (a) and baPWV (b) in patients with type 2 diabetes.

CAVI also decreased significantly after meal intake $(1652 \pm 286-$ $1586 \pm 240 \mathrm{~cm} \mathrm{~s}^{-1} ; P=0.002,9.52 \pm 0.92-9.20 \pm 0.89 ; P=0.037$, respectively) (Figures $1 \mathrm{~b}$ and $\mathrm{c}$ ). Positive linear correlations were observed between baPWV and CAVI in both fasting $(r=0.676, P=0.003)$ and postprandial states $(r=0.614, P=0.009)$. On the other hand, no significant association was observed between AI and other pulse wave indices. The percent change of AI from fasting to 60 and $120 \mathrm{~min}$ after meal intake was $-12.6 \pm 8.3$ and $-7.8 \pm 7.1 \%$, respectively. The percent change of baPWV and CAVI from fasting to postprandial state was $-3.7 \pm 3.7$ and $-3.2 \pm 5.9 \%$, respectively.

\section{Correlation between $\Delta$ pulse wave indices and clinical variables}

Linear regression analysis was performed to evaluate the relationship between $\Delta$ pulse wave indices and clinical variables. $\Delta_{60}$ (value $60 \mathrm{~min}$ after meal intake-fasting value) AI showed significant correlation between age ( $r=-0.706, P=0.002)$, body weight $(r=0.533, P=0.028)$, HbAlc $(r=-0.530, P=0.029)$ and $\Delta_{60} \operatorname{SBP}(r=0.528, P=0.029)$. In contrast, $\Delta_{60} \mathrm{AI}$ was not correlated with $\Delta_{60}$ glucose $(r=0.010$, $P=0.971)$ and $\Delta_{60}$ insulin $(r=0.202, P=0.437)$. Stepwise multiple regression analysis among these correlating variables revealed that age and HbAlc were the independent determinants of $\Delta_{60}$ AI $\left(F=13.9, R^{2}=0.665\right) . \Delta_{120}$ (value $120 \mathrm{~min}$ after meal intake-fasting value) AI showed significant linear correlation between age ( $r=-0.620, P=0.008)$, body weight $(r=0.604, P=0.010), \Delta_{120}$ SBP $(r=0.725, \quad P=0.001), \Delta_{120}$ DBP $(r=0.656, P=0.004), \Delta_{120}$ pulse pressure $(r=0.520, P=0.033), \Delta_{120}$ heart rate $(r=-0.841, P<0.001)$ and fasting $\mathrm{AI}(r=-0.519, P=0.033)$ (Figure 2a). $\Delta_{120} \mathrm{AI}$ showed no significant correlation with $\Delta_{120}$ glucose $(r=0.106, P=0.685)$ and $\Delta_{120}$ insulin $(r=-0.411, P=0.101)$. Stepwise multiple regression analysis showed that age, $\Delta_{120} \mathrm{DBP}$ and $\Delta_{120}$ heart rate were the independent determinants of $\Delta_{120} \mathrm{AI}\left(F=29.3, R^{2}=0.871\right)$. In addition, $\Delta$ baPWV was significantly correlated with HbAlc $(r=-0.549, P=0.023), \Delta$ SBP $(r=0.584, P=0.014), \Delta$ pulse pressure $(r=0.623, P=0.008)$ and fasting baPWV $(r=-0.717, P=0.001)$ (Figure 2b). $\Delta$ baPWV was not correlated with $\Delta_{60}$ glucose $(r=-0.218, P=0.401), \Delta_{120}$ glucose $(r=-0.312, P=0.223), \Delta_{60}$ insulin $(r=0.345, P=0.176)$ and $\Delta_{120}$ insulin $(r=0.128, P=0.625)$. Stepwise multiple regression analysis showed that fasting baPWV and $\Delta$ pulse pressure were the independent determinants of $\Delta$ baPWV $\left(F=17.0, R^{2}=0.708\right)$. In contrast, linear regression analysis showed no significant correlation between $\Delta$ CAVI and any clinical variables.

\section{DISCUSSION}

The results of this study show that AI, baPWV and CAVI decreased significantly after meal intake in patients with type 2 diabetes.

Although the precise mechanisms behind the postprandial decreases in these indices are unclear, earlier studies have shown several changes after meal ingestion. Meal ingestion leads to biochemical and hormonal changes including secretion of gastric acid and gut peptides. Increased insulin acts as a vasodilator through generation of nitric oxide. ${ }^{20}$ Splanchnic blood flow is enhanced by a variety of postprandial hemodynamic changes including an increase in heart rate, stroke volume and cardiac output. ${ }^{21,22}$ Sympathetic nerve activity increases in response to food ingestion to maintain blood pressure in healthy persons. ${ }^{23}$ On the other hand, an inadequate sympathetic response sometimes occurs in elderly persons and patients with autonomic neuropathy, ${ }^{24}$ and results in a decrease in blood pressure. 
Among these changes, our results indicate that hemodynamic changes have important functions in the decrease in AI and baPWV. In this study, $\Delta$ baPWV is correlated with changes of hemodynamic variables such as $\Delta \mathrm{SBP}$ and $\Delta$ pulse pressure. $\Delta \mathrm{AI}$ is also correlated with a variety of hemodynamic changes such as SBP, DBP, pulse pressure and heart rate. These results are consistent with earlier studies showing both baPWV and AI as dependent indices on hemodynamic variables such as blood pressure and heart rate. ${ }^{25-28}$ On the other hand, in this study, the degree of postprandial changes in glucose and insulin levels were not correlated with $\Delta$ baPWV and $\Delta$ AI. This finding is also consistent with an earlier study showing decrease in AI under the insulin clamp technique, whereas no significant relation between $\Delta \mathrm{AI}$ and changes in glucose or insulin levels. ${ }^{29}$ These results support the hypothesis that postprandial hemodynamic changes may affect baPWV and AI more directly than the other changes including biochemical, hormonal and autonomic changes.

With regard to postprandial changes in CAVI, we found no correlated clinical variable including postprandial hemodynamic changes. As CAVI was developed as an index of arterial stiffness without the influence of blood pressure, ${ }^{8,30-32}$ postprandial hemodynamic effects may be limited for CAVI. Further investigation should be addressed to clarify the influence of biochemical, hormonal or autonomic factors in postprandial changes in CAVI.

The caloric value of the test meal used in this study was $500 \mathrm{kcal}$, an amount considered to provide appropriate energy for a daily meal. On the other hand, $28 \mathrm{~g}$ of fat occupied about $50 \%$ of total calorie intake. Berry et al. revealed impaired endothelial function after $50 \mathrm{~g}$ of highfat diet. ${ }^{33}$ In addition, they also showed different effects on postprandial endothelial function after stearic acid- or oleic acid-rich fat loading even though total calorie intake was similar in the two meals. Therefore, endothelial dysfunction may influence postprandial changes in arterial stiffness. However, our earlier study did not show significant changes in flow-mediated dilatation after $24 \mathrm{~g}$ of fat loading, ${ }^{34}$ suggesting that the amount of fat loaded in this study has little influence on endothelial function. In addition, Raitakari et al. showed that high-fat (61 g; rich in saturated or monounsaturated fats) meal loading did not lead to endothelial dysfunction, as assessed by flow-mediated dilatation in the brachial artery, but it did induce vasodilatation and increased resting and post-ischemic-stimulated forearm blood flow. ${ }^{35}$ Consequently, several issues are still in question; namely, the relationship between postprandial changes in arterial stiffness and endothelial function, the effects of different meal composition such as high-fat or carbohydrate meals on postprandial changes in arterial reactivity. Therefore, further investigation should be conducted to illuminate these unsolved issues. However, this study still carries a clear message that the pulse wave indices widely used in clinical practice significantly decrease after a daily meal intake in patients with type 2 diabetes, in spite of an ideal amount of calorie intake.

Our results showed that mean percent changes of baPWV and CAVI from fasting to postprandial state were $-3-4 \%$. These changes, on the whole, might be considered acceptable for the routine assessment of aortic stiffness in clinical practice. However, the maximal percent decreases of baPWV and CAVI reached -10.8 and $-14.1 \%$, respectively. In addition, mean percent change of AI showed a larger decrease during the postprandial state. Therefore, when assessing pulse wave indices, especially for AI in the postprandial state, caution should be taken not to underestimate arterial stiffness.

Patients with type 2 diabetes have an increased risk of atherosclerosis and cardiovascular mortality. On the other hand, postprandial hypotension has been observed in elderly patients with advanced atherosclerosis ${ }^{24,36,37}$ and in patients with autonomic neuropathy, ${ }^{24,38,39}$ which are frequently seen among patients with diabetes. Unfortunately, we did not assess autonomic function such as heart rate variability. However, the degree of SBP reduction both 60 and $120 \mathrm{~min}$ after meal intake $(11.8 \pm 9.8$ and $10.8 \pm 10.3 \mathrm{~mm} \mathrm{Hg}$, respectively) was much larger compared with our recent study, which used the same amount of CalorieMate ${ }^{13}$ and with another study by Ahuja et al. ${ }^{40}$ This might imply the presence of sympathetic nerve dysfunction in some of the patients with type 2 diabetes we studied.

Interestingly, our results revealed that the degree of postprandial changes in $\mathrm{AI}$ and baPWV correlated with those fasting levels. That is, in a case with advanced aortic stiffness, meal ingestion may result in larger hemodynamic changes and may contribute to larger decreases in $\mathrm{AI}$ and baPWV in patients with type 2 diabetes.

This study has several limitations. First, we cannot exclude the possibility that certain medications may affect these pulse indices. To reduce this limitation, however, we provided the withdrawal periods of antihypertensive and lipid-lowering agents. Second, because this study did not have healthy control subjects or patients without diabetes, it remains uncertain as to whether decrease of these pulse indices after meal intake would also be present in healthy persons or in patients without diabetes. Third, the study population was relatively small. However, our results are reasonable given that postprandial changes in $\mathrm{AI}$ and baPWV are dependent on hemodynamic changes, whereas CAVI is influenced less by hemodynamic changes.

In conclusion, AI, baPWV and CAVI significantly decreased after daily meal intake in patients with type 2 diabetes. Changes in AI and baPWV but not CAVI after meal intake were influenced by postprandial hemodynamic changes.

\section{CONFLICT OF INTEREST}

The authors declare no conflict of interest.

\section{ACKNOWLEDGEMENTS}

We are grateful to Saori Shimizu, Saori Nagatoshi and Mari Oishi for their assistance in measurements of baPWV and CAVI.

1 Blacher J, Asmar R, Djane S, London GM, Safar ME. Aortic pulse wave velocity as a marker of cardiovascular risk in hypertensive patients. Hypertension 1999; 33: 1111-1117.

2 Laurent S, Boutouyrie P, Asmar R, Gautier I, Laloux B, Guize L, Ducimetiere P, Benetos A. Aortic stiffness is an independent predictor of all-cause and cardiovascular mortality in hypertensive patients. Hypertension 2001; 37: 1236-1241.

3 Cruickshank K, Riste L, Anderson SG, Wright JS, Dunn G, Gosling RG. Aortic pulsewave velocity and its relationship to mortality in diabetes and glucose intolerance: an integrated index of vascular function? Circulation 2002; 106: 2085-2090.

4 Blacher J, Guerin AP, Pannier B, Marchais SJ, Safar ME, London GM. Impact of aortic stiffness on survival in end-stage renal disease. Circulation 1999; 99: 2434-2439.

5 Guerin AP, Blacher J, Pannier B, Marchais SJ, Safar ME, London GM. Impact of aortic stiffness attenuation on survival of patients in end-stage renal failure. Circulation 2001; 103: 987-992.

6 Kubo T, Miyata M, Minagoe S, Setoyama S, Maruyama I, Tei C. A simple oscillometric technique for determining new indices of arterial distensibility. Hypertens Res 2002; 25: 351-358.

7 Yamashina A, Tomiyama H, Takeda K, Tsuda H, Arai T, Hirose K, Koji Y, Hori S, Yamamoto $Y$. Validity, reproducibility, and clinical significance of noninvasive brachialankle pulse wave velocity measurement. Hypertens Res 2002; 25: 359-364.

8 Shirai K, Utino J, Otsuka K, Takata M. A novel blood pressure-independent arterial wall stiffness parameter; cardio-ankle vascular index (CAVI). J Atheroscler Thromb 2006; 13: 101-107.

9 Okura T, Watanabe S, Kurata M, Manabe S, Koresawa M, Irita J, Enomoto D, Miyoshi K, Fukuoka T, Higaki J. Relationship between cardio-ankle vascular index (CAVI) and carotid atherosclerosis in patients with essential hypertension. Hypertens Res 2007; 30: 335-340.

10 Izuhara M, Shioji K, Kadota S, Baba O, Takeuchi Y, Uegaito T, Mutsuo S, Matsuda M. Relationship of cardio-ankle vascular index (CAVI) to carotid and coronary arteriosclerosis. Circ J 2008; 72: 1762-1767. 
11 Takazawa K, Kobayashi H, Shindo N, Tanaka N, Yamashina A. Relationship between radial and central arterial pulse wave and evaluation of central aortic pressure using the radial arterial pulse wave. Hypertens Res 2007; 30: 219-228.

12 Munir S, Guilcher A, Kamalesh T, Clapp B, Redwood S, Marber M, Chowienczyk P. Peripheral augmentation index defines the relationship between central and peripheral pulse pressure. Hypertension 2008; 51: 112-118.

13 Funada J, Takata Y, Hashida H, Matsumoto Y, Sato S, Hiasa G, Inoue K, Higaki J, Okayama $\mathrm{H}$. Dysfunctional central hemodynamic regulation after daily meal intake in metabolic syndrome. Atherosclerosis (e-pub ahead of print 10 November 2009).

14 Murgo JP, Westerhof N, Giolma JP, Altobelli SA. Aortic input impedance in normal man: relationship to pressure wave forms. Circulation 1980; 62: 105-116.

15 Kelly R, Hayward C, Avolio A, O'Rourke M. Noninvasive determination of age-related changes in the human arterial pulse. Circulation 1989; 80: 1652-1659.

16 Sako H, Miura S, Kumagai K, Saku K. Associations between augmentation index and severity of atheroma or aortic stiffness of the descending thoracic aorta by transesophageal echocardiography. Circ J 2009; 73: 1151-1156.

17 Weber T, Auer J, O'Rourke MF, Kvas E, Lassnig E, Berent R, Eber B. Arterial stiffness, wave reflections, and the risk of coronary artery disease. Circulation 2004; 109: 184-189.

18 Haffner SM, Lehto S, Rönnemaa T, Pyörälä K, Laakso M. Mortality from coronary heart disease in subjects with type 2 diabetes and in nondiabetic subjects with and without prior myocardial infarction. N Engl J Med 1998; 339: 229-234.

19 Expert Committee on the Diagnosis and Classification of Diabetes Mellitus. Report of the Expert Committee on the Diagnosis and Classification of Diabetes Mellitus. Diabetes Care 1997; 20: 1183-1197.

20 Scherrer U, Randin D, Vollenweider P, Vollenweider L, Nicod P. Nitric oxide release accounts for insulin's vascular effects in humans. J Clin Invest 1994; 94: 2511-2515.

21 Lipsitz LA, Ryan SM, Parker JA, Freeman R, Wei JY, Goldberger AL. Hemodynamic and autonomic nervous system responses to mixed meal ingestion in healthy young and old subjects and dysautonomic patients with postprandial hypotension. Circulation 1993; 87: $391-400$

22 Mathias CJ. Postprandial hypotension. Pathophysiological mechanisms and clinical implications in different disorders. Hypertension 1991; 18: 694-704.

23 Berne C, Fagius J, Niklasson F. Sympathetic response to oral carbohydrate administration. Evidence from microelectrode nerve recordings. J Clin Invest 1989; 84: 1403-1409.

24 Mathias CJ, da Costa DF, Fosbraey P, Bannister R, Wood SM, Bloom SR, Christensen NJ. Cardiovascular, biochemical and hormonal changes during food-induced hypotension in chronic autonomic failure. J Neurol Sci 1989; 94: 255-269.

25 Matsui Y, Kario K, Ishikawa J, Eguchi K, Hoshide S, Shimada K. Reproducibility of arterial stiffness indices (pulse wave velocity and augmentation index) simultaneously assessed by automated pulse wave analysis and their associated risk factors in essential hypertensive patients. Hypertens Res 2004; 27: 851-857.
26 Kubozono T, Miyata M, Ueyama K, Nagaki A, Otsuji Y, Kusano K, Kubozono O, Tei C. Clinical significance and reproducibility of new arterial distensibility index. Circ J 2007; 71: 89-94.

27 Hayward CS, Kelly RP. Gender-related differences in the central arterial pressure waveform. J Am Coll Cardiol 1997; 30: 1863-1871.

28 McEniery CM, Yasmin, Hall IR, Qasem A, Wilkinson IB, Cockcroft JR, ACCT Investigators. Normal vascular aging: differential effects on wave reflection and aortic pulse wave velocity: the Anglo-Cardiff Collaborative Trial (ACCT). J Am Coll Cardiol 2005; 46: 1753-1760.

29 Tamminen M, Westerbacka J, Vehkavaara S, Yki-Järvinen H. Insulin-induced decreases in aortic wave reflection and central systolic pressure are impaired in type 2 diabetes. Diabetes Care 2002; 25: 2314-2319.

30 Ibata J, Sasaki H, Kakimoto T, Matsuno S, Nakatani M, Kobayashi M, Tatsumi K, Nakano Y, Wakasaki H, Furuta H, Nishi M, Nanjo K. Cardio-ankle vascular index measures arterial wall stiffness independent of blood pressure. Diabetes Res Clin Pract 2008; 80: 265-270.

31 Takaki A, Ogawa H, Wakeyama T, Iwami T, Kimura M, Hadano Y, Matsuda S, Miyazaki Y, Hiratsuka A, Matsuzaki M. Cardio-ankle vascular index is superior to brachialankle pulse wave velocity as an index of arterial stiffness. Hypertens Res 2008; 31 : 1347-1355.

32 Satoh N, Shimatsu A, Kato Y, Araki R, Koyama K, Okajima T, Tanabe M, Ooishi M, Kotani K, Ogawa Y. Evaluation of the cardio-ankle vascular index, a new indicator of arterial stiffness independent of blood pressure, in obesity and metabolic syndrome. Hypertens Res 2008; 31: 1921-3190.

33 Berry SE, Tucker S, Banerji R, Jiang B, Chowienczyk PJ, Charles SM, Sanders TA. Impaired postprandial endothelial function depends on the type of fat consumed by healthy men. J Nutr 2008; 138: 1910-1914.

34 Funada J, Sekiya M, Hamada M, Hiwada K. Postprandial elevation of remnant lipoprotein leads to endothelial dysfunction. Circ J 2002; 66: 127-132.

35 Raitakari OT, Lai N, Griffiths K, McCredie R, Sullivan D, Celermajer DS. Enhanced peripheral vasodilation in humans after a fatty meal. J Am Coll Cardiol 2000; 36: 417-422.

36 Westenend M, Lenders JW, Thien T. The course of blood pressure after a meal: a difference between young and elderly subjects. J Hypertens 1985; 3 (Suppl 3): S417-S419.

37 Lipsitz LA, Fullerton KJ. Postprandial blood pressure reduction in healthy elderly. J Am Geriatr Soc 1986; 34: 267-270.

38 Robertson D, Wade D, Robertson RM. Postprandial alterations in cardiovascular hemodynamics in autonomic dysfunction states. Am J Cardiol 1981; 48: 1048-1052.

39 Jansen RW, Lipsitz LA. Postprandial hypotension: epidemiology, pathophysiology, and clinical management. Ann Intern Med 1995; 122: 286-295.

40 Ahuja KD, Robertson IK, Ball MJ. Acute effects of food on postprandial blood pressure and measures of arterial stiffness in healthy humans. Am J Clin Nutr 2009; 90: 298-303. 